\title{
Frangi Goes US : Multiscale Tubular Structure Detection Adapted to 3D Ultrasound
}

\author{
Paulo Waelkens, Seyed-Ahmad Ahmadi, and Nassir Navab \\ Computer Aided Medical Procedures, Technische Universität München, Germany
}

\begin{abstract}
We propose a Hessian matrix based multiscale tubular structure detection (TSD) algorithm adapted to 3D B-mode vascular US images. The algorithm is designed to highlight blood vessel centerline points and yield an estimate of the cross-section radius at each centerline point. It can be combined with a simple centerline extraction scheme, yielding precise, fast and fully automatic lumen segmentation initializations.

TSD algorithms designed with CTA and MRA datasets in mind, e.g. the Frangi Filter [3], are not capable of reliably distinguishing centerline points from other points in vascular US datasets, since some assumptions underlying these algorithms are not reasonable for US datasets. The algorithm we propose, does not have these shortcomings and performs significantly better on vascular US datasets.

We propose a statistic to evaluate how well a TSD algorithm is able to distinguish centerline points from other points. Based on this statistic, we compare the Frangi Filter to various versions of our new algorithm, on 11 3D US carotid datasets.
\end{abstract}

\section{Introduction and Medical Motivation}

In recent years, tracked freehand ultrasound (3D US) emerged as a viable and low-cost (compared to CT and MRI) 3D imaging modality. 3D US shows a lot of potential, especially for vascular imaging (e.g. of the carotid arteries).

Today, when considering serious procedures, e.g. a carotid endarterectomy, vascular surgeons request CT or MRI angiographies (CTA and MRA) 1 to get a clear 3D visualization of the diseased vascular tree. However, angiographies have serious drawbacks: they are expensive, time consuming and present health hazards, in particular for elderly patients.

If it becomes possible to automatically and reliably extract more clinically relevant information from vascular 3D US, one would expect the need for angiographies to decline. We hope the TSD algorithm proposed in this paper is a first step in this direction.

Characteristics of Vascular US. In figure 1, left column, an US image of the cross-section of a common carotid artery is shown. The vessel lumen cross-section

${ }^{1}$ Intravascular ultrasound (IVUS) is not used for carotid imaging, as the US probe might rupture atheromas. We thus do not consider it an alternative to CTA/MRA. 
can be identified by its size, shape (roughly circular), signal response (uniformly low) and surroundings (a narrow ring of higher signal response points). One can also notice the influence the US beam direction has on the US signal response strength: edges orthogonal to the beam direction reflect the sound waves better (leading to a higher response) than edges with other orientations. The closer the edge comes to being parallel to the beam direction, the lower the reflection and the US response will be. During a freehand US scan, the practitioner has limited possibilities of where to locate and how to orient the probe. Thus, usually only a few beam directions can be used to generate the US response of a given voxel, and a strong direction dependency will be present in the reconstructed $3 \mathrm{D}$ dataset, in contrast to CT and MRI (and IVUS) datasets.

This is why, whilst assuming the symmetry of vessel features around centerlines is reasonable in CTA and MRA, the same does not apply for 3D US datasets.

Related Works. Guerrero et al [4] and Carvalho et al [2] propose centerline detection and tracking algorithms for sequences of tracked $2 \mathrm{D}$ vascular freehand US images. On each image they attempt to fit an ellipse to the vessel crosssection, and connect this $2 \mathrm{D}$ information with the spatial information provided by the tracking system. In [2], the direction dependency of US images is considered. Their approaches are inherently 2D, while we focus on less restrictive 3D approaches.

Among 3D algorithms, we point especially to the Frangi Filter, a multiscale TSD algorithm proposed by Frangi in [3. Works published by Krissian et al, e.g. [5], and Pock et al, e.g. [7], among others, present similar and related ideas. All these authors use the information provided by the Hessian matrix of the smoothed data: Frangi proposes a vesselness measure as a function of the eigenvalues of the Hessian matrix; Krissian et al propose to combine the Hessian matrix with the structure tensor, and base their measure on the eigenvalues and eigenvectors of this combined descriptor; Pock et al propose a medialness function, which is evaluated on the plane spanned by the two largest principal curvatures of the Hessian matrix. To normalize their results over multiple scales, the authors of [3], 5] and [7 point to the scale-space theory proposed by Lindeberg [6], i.e. smoothing with Gaussian kernels and using $\gamma$-normalized partial derivatives.

To the best of our knowledge, the TSD algorithms proposed by all cited authors assume symmetry of features around centerlines.

We focus only on multiscale Hessian based approaches, as they allow the automatic detection of an arbitrary number of vessels, of arbitrary lengths and orientations over multiple scales; in contrast to more restrictive approaches like statistical shape models, or semi-automatic methods (e.g. Aylward et al [1]).

Contributions. We propose a Hessian matrix based multiscale tubular structure detection (TSD) algorithm adapted to 3D B-mode vascular US images. Without loss of generality (WLOG), we sort the Hessian's eigenvalues $\left|\lambda_{1}\right| \leq$ $\left|\lambda_{2}\right| \leq\left|\lambda_{3}\right|$. WLOG the associated eigenvectors $v_{1}, v_{2}$ and $v_{3}$ are normalized: $\left\|v_{i}\right\|=1$. 
We propose 4 changes, which will be described in more detail in the next section, on Frangi's vesselness algorithm:

- Ideal lumen model and associated kernel: We propose the use of a nonGaussian smoothing kernel, whose second partial derivatives better fit the expected lumen shape

- Beam direction dependency: At a centerline point, we assume the direction of highest curvature $\left(=v_{3}\right)$ will be parallel to the US beam direction

- Intensity prior: We integrate the information that the vessel interior, i.e. the neighborhood of a vessel centerline point, has low US signal response

- Vessel feature assymetry: At a centerline point, we expect $\frac{\left|\lambda_{2}\right|}{\left|\lambda_{3}\right|}<1$, i.e. we expect assymetry of the depicted vessel features and compensate for this fact

\section{Materials and Methods}

Basic Algorithm for $\boldsymbol{C T A}$ and $\boldsymbol{M R A}$. We describe the basic workings of the Frangi Filter. WLOG assume a dark lumen surrounded by brighter tissue. Consider the Hessian matrix of the smoothed data at a point $p$,

$$
\operatorname{smoothData}_{r}(p):=\left(\operatorname{kernel}\left(\zeta_{r}\right) * \operatorname{data}\right)(p)
$$

where $\zeta_{r}$ is the kernel parameter, a function of the scale parameter $r$. Recall that $\lambda_{i}$ corresponds to the second directional derivative of the smoothed data in the direction $v_{i}(i=\{1,2,3\})$, and $\left\{v_{1}, v_{2}, v_{3}\right\}$ is an orthonormal basis of $\mathbb{R}^{3}$. If $p$ is a lumen centerline point with cross-section radius $r$, Frangi assumes

$$
\begin{array}{rr}
\lambda_{1} \approx 0 & \operatorname{sign}\left(\lambda_{2}\right)=\operatorname{sign}\left(\lambda_{3}\right)=1 \\
\left|\lambda_{2}\right|>>0 & \lambda_{2}=\lambda_{3}
\end{array}
$$

i.e. at a centerline point, the curvature of the smoothed data in the directions $v_{2}$ and $v_{3}$ is large, and the curvature in the direction $v_{1}$ is small. $v_{2}$ and $v_{3}$ span the cross-section plane and $v_{1}$ is the centerline tangent direction.

He proposes a vesselness measure that is higher at points where his assumptions are satisfied. The assumption $\lambda_{2}=\lambda_{3}$ is not reasonable for US datasets, due to direction dependency. Also, only the Gaussian kernel is considered for multiscale smoothing, following the scale-space theory proposed by Lindeberg [6].

Ideal Lumen Model. We assume an idealized model for the vessel lumen in our framework. The lumen is represented by a dark cylinder (in 3D) or a dark disc (in $2 \mathrm{D}$ cross-sections) of a given radius $r$. The lumen is surrounded by a white ring of a given thickness $t h(r)$, which represents the vessel wall's echo-response and echogenicities in close proximity to the vessel. Based on the observation of $163 \mathrm{D}$ US carotid datasets, we chose $t h(r)=0.6 r$. The tissue outside the white ring can have arbitrary response patterns and is thus represented by noise. The idealized model is illustrated in figure 1. It serves two purposes:

1) it provides a model of the shape that the algorithm is expected to find, i.e. it allows us to build an adequate smoothing convolution kernel and 
2) the model is used to provide a reference vesselness value for centerline points of tubular structures at the scale $r$; for the scale $r$, this value is defined as the vesselness value at a centerline point of the ideal model for this scale.

Multiscale Normalization Scheme. For each scale $r_{i}$ the algorithm iterates over, we obtain the reference vesselness value. Vesselness results for the scale $r_{i}$ are normalized (i.e. divided) by the associated reference value.

Design of the Smoothing Kernel. At the appropriate scale $r, \lambda_{2}$ and $\lambda_{3}$ should be high at a vessel's centerline point $c$. Thus, based on the ideal lumen model for the scale $r$, $i \operatorname{Lum}(r)$, the kernel should be selected so that

$$
\left[\frac{\partial^{2}}{\partial k^{2}}\left(\operatorname{kernel}\left(\zeta_{r}\right) * i \operatorname{Lum}(r)\right)\right](c)=\left[\left(\frac{\partial^{2}}{\partial k^{2}} \operatorname{kernel}\left(\zeta_{r}\right)\right) * i \operatorname{Lum}(r)\right](c)
$$

is as large as possible, where $k$ is the second or third coordinate in the coordinate system $\left\{v_{1}, v_{2}, v_{3}\right\}$ (i.e. expression (1) $=\lambda_{2}$ or $\lambda_{3}$ ). In particular, the expression should be larger at centerline points than at all other points.

Additionally, an acceptable smoothing kernel must be spherically symmetric, unimodal, non-negative and add up to 1 . It follows that the first and second partial derivatives of the kernel add to 0 .

By symmetry it suffices to define the kernel on a half-line starting from 0 . Let $\mathbb{1}_{S}$ denote the indicator function of a set $S$. Notice from equation (1) that convolutions and differentiations commute. Intuitively, an ideal kernel would be defined by the function $g: \mathbb{R}_{+} \rightarrow \mathbb{R}_{+}$, where

$$
\frac{d^{2}}{d t^{2}} g(t ; r, t h)=\frac{1}{K}\left[-\mathbb{1}_{\{0 \leq t<r\}}+\frac{r}{t h} \mathbb{1}_{\{r \leq t<(r+t h)\}}\right]
$$

and $K>0$. The one dimensional kernel generated, $k e r_{g}: \mathbb{R} \rightarrow \mathbb{R}_{+}$, would be:

$$
k e r_{g}(t ; r, t h)=\mathbb{1}_{\{t<0\}} g(-t ; r, t h)+\mathbb{1}_{\{t \geq 0\}} g(t ; r, t h)
$$

$K$ is chosen so $k e r_{g}$ adds up to 1 . At a centerline point, $\frac{d^{2}}{d t^{2}} k e r_{g}$ gives negative weights to the lumen, positive weights to the white ring and ignores the noise: an ideal match to the ideal lumen model in one dimension. However, $g$ is not a smooth function. For performance reasons, convolutions in $\mathbb{R}^{3}$ are done in the frequency domain. To avoid aliasing, all functions involved should be smooth. So, instead of $g$, we use a smooth function of similar shape, $g^{*}: \mathbb{R}_{+} \rightarrow \mathbb{R}_{+}$:

$$
g^{*}\left(t ; \zeta_{r}\right)=\frac{1}{K} e^{P_{12}^{*}\left(t ; \zeta_{r}\right)}
$$

Where the polynomial $P_{12}^{*}$ is given by

$$
\begin{gathered}
P_{12}^{*}\left(t ; \zeta_{r}\right)=-0.5\left(c_{12}\left(\frac{t \cdot 0.408}{\zeta_{r}}\right)^{12}+c_{10}\left(\frac{t \cdot 0.408}{\zeta_{r}}\right)^{10}+c_{8}\left(\frac{t \cdot 0.408}{\zeta_{r}}\right)^{8}\right. \\
\left.+c_{6}\left(\frac{t \cdot 0.408}{\zeta_{r}}\right)^{6}+c_{4}\left(\frac{t \cdot 0.408}{\zeta_{r}}\right)^{4}+c_{2}\left(\frac{t \cdot 0.408}{\zeta_{r}}\right)^{2}\right)
\end{gathered}
$$


with $c_{12}=1, c_{10}=409.6, c_{8}=128, c_{6}=42.66, c_{4}=16$ and $c_{2}=8$.

The generated $\mathbb{R}^{3}$ kernel is given by:

$$
\operatorname{kernel}^{*}\left(t ; \zeta_{r}\right)=\frac{1}{\left|e^{P_{12}^{*}\left(t ; \zeta_{r}\right)}\right|_{1}} e^{P_{12}^{*}\left(t ; \zeta_{r}\right)}
$$

where $t$ is the distance from the origin (recall: spherical symmetry) and $|\cdot|_{1}$ denotes the $\mathscr{L}^{1}$ norm. To obtain the link between $\zeta_{r}$ and $r$ : fix a positive value of $r^{*}$ (e.g. $r^{*}=1$ ) and find $\zeta^{*}$ that maximizes

$$
\max _{\zeta^{*}>0} \frac{1}{\left|\frac{\partial^{2}}{\partial k^{2}} \operatorname{kernel}\left(\zeta^{*}\right)\right|_{1}}\left(\frac{\partial^{2}}{\partial k^{2}} \operatorname{kernel}\left(\zeta^{*}\right) * i \operatorname{Lum}\left(r^{*}\right)\right)(c)
$$

where $k$ is the second or third coordinate in the coordinate system $\left\{v_{1}, v_{2}, v_{3}\right\}$. The link will be given by

$$
\zeta_{r}=\frac{\zeta^{*}}{r^{*}} r
$$

Expression (6) is maximized for the kernel parameter $\zeta^{*}$ that minimizes the proportion of wrong associations of $\frac{\partial^{2}}{\partial k^{2}} \operatorname{kernel}\left(\zeta^{*}\right)$ values to vessel features, i.e. when the proportion of low and negative weights for white ring voxels is kept at a minimum. For the 3D version of kernel $^{*}$ we numerically obtained $\zeta_{r}=0.95 r$. For the 3D Gaussian kernel, we numerically obtained $\left(\sigma_{r}=\right) \zeta_{r}=0.575 r$.

Notice $g$ has 2 parameters to define its size, $r$ and $t h$, whilst $g^{*}$ only has $\zeta_{r}$. $g^{*}$ was designed so that $\frac{d^{2}}{d t^{2}} g^{*}$ fit $\frac{d^{2}}{d t^{2}} g$ with $t h=0.6 r$. For thicker walls choose polynomials of lower degree (e.g. Gaussian kernel); for narrower walls, higher.

The Gaussian kernel only has 1 parameter, $\sigma_{r}$, to define its size, which implies it fits $g$ for a specific ratio $\frac{t h}{r}: t h(r) \approx 3 r$. This does not match the selected ideal lumen model as well as kernel ${ }^{*}$. The Gaussian kernel $\frac{d^{2}}{d t^{2}}$ either gives significant importance to the noise component and possibly negative weights to white ring points, or, alternatively, associates low weights to white ring points, yielding low values of $\lambda_{2}$ and $\lambda_{3}$ at $c$. In short, the Gaussian is not always the ideal choice as smoothing kernel for Hessian-based algorithms. Figure 1 illustrates this fact:

Beam Direction Dependency. Due to the physics behind US imaging, the ultrasound echo reponse will be higher at vessel wall sections which are oriented perpendicularly to the US beam than at vessel wall sections with different orientations, since sections perpendicular to the US beam reflect more of it directly back to the emitter (this effect is visible on the US image depicted in figure 1). For a lumen centerline point, this fact translates to the highest curvature being in the US beam direction. Thus, at a centerline point, we expect the direction of highest curvature (by definition, the eigenvector $v_{3}$ ) to be parallel to the US beam. To evaluate if this assumption is met, we propose the measure:

$$
B D D=\mid<v_{3}, \text { beamDirection }>\mid \in[0 ; 1]
$$



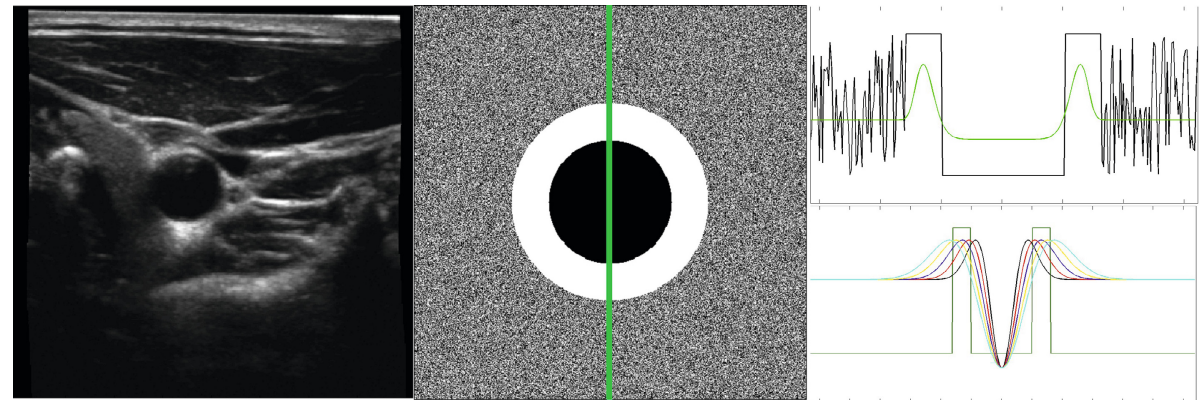

Fig. 1. Left: Common carotid artery cross-section; Middle: Ideal lumen cross-section with line segment overlaid; Right: Line segment of ideal lumen cross-section with $\frac{\partial^{2}}{\partial k^{2}}$ kernel overlaid: proposed kernel (top, in green), multiple attempts to fit the gaussian kernel (bottom; noise removed for better visualization)

Intensity Prior. We assume that the vessel lumen has a uniformly low US signal response (=dark), and is surrounded by higher signal response regions(=bright). For a given lumen radius $r$, the data smoothed with a Gaussian kernel with, e.g., $\sigma=5 r$, smoothData $\operatorname{DP}_{P}$ (IP = intensity prior) will still be dark at a centerline point, but brighter at lumen points closer to the lumen boundaries. So, to better distinguish centerline points from other lumen points, we propose the measure:

$$
i \text { Prior }=\frac{1}{1+i p \cdot \operatorname{smoothData} \operatorname{IP}_{P}}
$$

where $i p \geq 0$ guides this measure's importance. An added advantage is that bright regions, even with high values of $\lambda_{2}$ and $\lambda_{3}$, are also penalized.

Vessel Feature Assymetry. For the reasons described in section "Beam direction dependency", at a centerline point we also expect $\lambda_{2}<\lambda_{3}$. But, we still expect $\frac{\lambda_{2}}{\lambda_{3}}>>0$. Thus, we set a threshold thresh quo for acceptable values of $\frac{\lambda_{2}}{\lambda_{3}}$ and propose the compensated eigenvalue quotient measure:

$$
c Q u o= \begin{cases}1 & \text { if } \frac{\left|\lambda_{2}\right|}{\left|\lambda_{3}\right|} \geq \text { thresh }_{\text {quo }} \\ \frac{\lambda_{2}}{\lambda_{3}} \text { otherwise. } & \end{cases}
$$

We estimate a value for thresh ${ }_{q u o}$ experimentally (see "Parameter selection").

Remaining Statistics. Similar to Frangi in [3, we use a blobness measure:

$$
R_{B}=\frac{\left|\lambda_{1}\right|}{\sqrt{\left|\lambda_{2} \lambda_{3}\right|}}
$$

and a measure of the relative significance of the information in the eigenvalues

$$
S=\sqrt{\left|\lambda_{2}\right|^{2}+\left|\lambda_{3}\right|^{2}}
$$


New Overall Vesselness Measure. Our modified vesselness measure is:

$$
\text { vess }= \begin{cases}0 & \text {,if }\left(\lambda_{2} \leq 0\right) \vee\left(\lambda_{3} \leq 0\right) \\ \text { iPrior } \cdot B D D^{\alpha} \cdot c Q u o^{\beta} \cdot e^{-\gamma R_{B}} \cdot\left(e^{\delta S}-1\right) & \text {,otherwise }\end{cases}
$$

\section{Experiments and Results}

Parameter selection $\alpha=1, \beta=1$ and $\gamma=1$. For numerical stability reasons, we take $\delta=0.1$. We choose ip so that the results are halved at very bright voxels: the data range is $[0,255]$, so we take $i p=\frac{1}{255}$.

thresh $h_{q u o}$, should be as high as possible, but low enough to yield good results at lumen centerline points. We perform supervised learning on 15 manually segmented cross-section planes from 5 3D US carotid datasets. For each plane, we choose the highest value of tresh $_{\text {quo }}$ that maximizes the vesselness at the marked centerline point. We test 26 equally spaced possible values of $\operatorname{tresh}_{\text {quo }}$ in $[0.01,1]$. The average threshold obtained was 0.3921 , the lowest threshold was 0.089 . We select the first quartile as the threshold value, i.e. thresh $_{\text {quo }}=0.2674$.

Comparison to Frangi Filter. We measure if the proposed modifications improve the algorithm's capability to distinguish centerline points (=high vesselness) from all other points. We manually segment 4 centerline points for each dataset and consider the statistic:

$$
\text { quality }=\frac{\sum_{i=1}^{4}\left|\operatorname{vess}\left(c_{i}\right)-\bar{X}_{\text {vess }}\right|}{\sigma_{\text {vess }}}
$$

where $c_{i}$ is a centerline point, $\bar{X}_{\text {vess }}$ is the average vesselness value and $\sigma_{\text {vess }}$ the standard deviation of the vesselness. Higher values of quality indicate a better capability to distinguish centerline points from the background.

The statistic is evaluated on 11 3D US carotid datasets. We mark 2 centerline points below and 2 above the bifurcation. We run 5 variations of the algorithm, adding a new feature for each variant: no modifications (as a proxy to the Frangi Filter); (+) the new kernel; (+) beam direction dependency; $(+)$ intensity prior; $(+)$ vessel feature assymetry. Results can be seen in figure 2 .

\section{Discussion and Conclusion}

We proposed a novel TSD algorithm adapted to US imaging properties, based on Frangi's Hessian matrix based multiscale vesselness algorithm. We identified which assumptions of Frangi's original formulation are unsuitable for vascular US, adapted them and added new assumptions based on prior knowledge of the expected lumen shape and of the physics underlying US imaging.

We also used a non-Gaussian smoothing kernel in the context of a multiscale algorithm. Our good results suggest that there are viable alternatives to the 


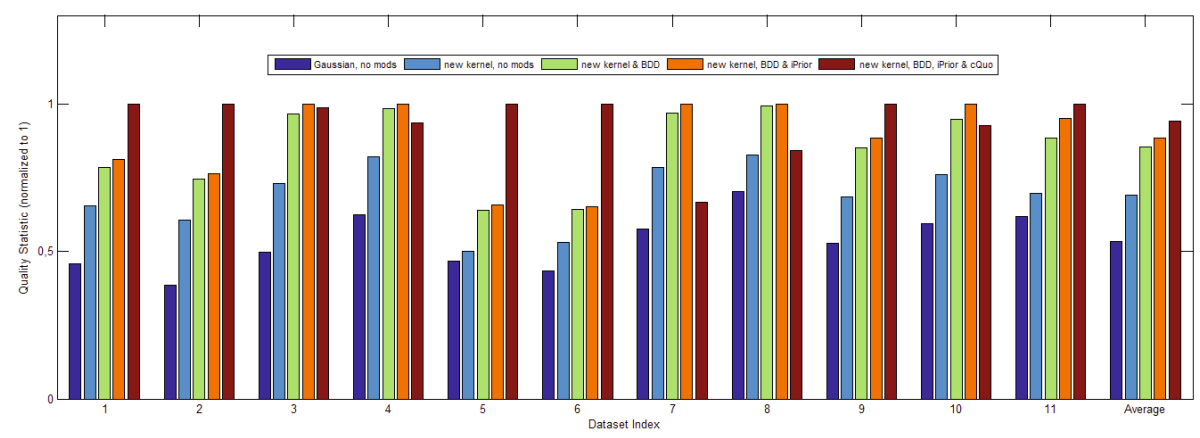

Fig. 2. quality statistic evaluated on 11 3D US carotid datasets; we tested 5 variations of the algorithm. Dark blue represents the results for the Frangi filter.

multiscale approach proposed in Lindeberg's work [6], especially if the expected shape of the feature of interest is already known.

All 4 modifications we proposed improved the algorithm's results, as measured by our quality statistic (see figure 2). The new kernel, the beam direction dependency and the intensity prior made the vesselness more stringent in general, while keeping the vesselness at centerline points unaltered. The vessel feature assymetry increased the vesselness at centerline points, but made the vesselness less stringent in general. While, on average, the results for this variant were better, significant decreases in the quality are possible (see datasets 7 and 8).

The algorithm can be parallelized extremely well. Using CUDA on a GTX 560 ti, the computation time for $256^{3}$ voxels was $<30$ secs, per scale.

As our promising results show, the suggested changes are important contributions to the knowledge on vessel detection in 3D US, bringing us closer to a robust, fast (with GPUs) and fully automatic vessel tree detection and segmentation initialization algorithm, for clinical applications in the future.

\section{References}

1. Aylward, S., Bullitt, E.: Initialization, noise, singularities, and scale in height ridge traversal for tubular object centerline extraction. IEEE Transactions on Medical Imaging 21(2), 61-75 (2002)

2. Carvalho, D.D., Klein, S., Akkus, Z., ten Kate, G.L., Schinkel, A.F., Bosch, J.G., van der Lugt, A., Niessen, W.J.: Estimating 3D lumen centerlines of carotid arteries in free-hand acquisition ultrasound. Int. J. Comput. Assist. Radiol. Surg. 7(2), 207-215 (2012)

3. Frangi, A.F.: Three-Dimensional Model-Based Analysis of Vascular and Cardiac Images. Ph.D. thesis, Universiteit Utrecht (2001)

4. Guerrero, J., Salcudean, S.E., McEwen, J.A., Masri, B.A., Nicolaou, S.: Real-time vessel segmentation and tracking for ultrasound imaging applications. IEEE Trans. Med. Imaging 26(8), 1079-1090 (2007) 
5. Krissian, K., Ellsmere, J., Vosburgh, K., Kikinis, R., Westin, C.F.: Multiscale Segmentation of the Aorta in 3D Ultrasound Images. In: 25th Annual Int. Conf. of the IEEE EMBS, pp. 638-641 (2003)

6. Lindeberg, T.: Scale-space theory: a basic tool for analyzing structures at different scales. Journal of Applied Statistics 21(1-2), 225-270 (1994)

7. Pock, T., Beichel, R., Bischof, H.: A Novel Robust Tube Detection Filter for 3D Centerline Extraction. In: Kalviainen, H., Parkkinen, J., Kaarna, A. (eds.) SCIA 2005. LNCS, vol. 3540, pp. 481-490. Springer, Heidelberg (2005) 